\title{
Peer-to-peer as an infrastructure service
}

\author{
Jiangchuan Liu $\cdot \mathrm{Ke} \mathrm{Xu} \cdot$ Yongqiang Xiong • \\ Dongchao Ma $\cdot$ Kai Shuang
}

Published online: 6 March 2014

(C) Springer Science+Business Media New York 2014

Peer-to-Peer (P2P) communication has attracted significant interest from both academia and industry, and has become a successful communication paradigm for diverse Internet applications, including file sharing, live streaming, and video on demand. Today P2P applications account for over $70 \%$ of the Internet traffic, and the percentage is still growing. P2P has also been used to facilitate the development of such new generation of applications as social networking and cloud computing. Many of these systems use P2P as an underlying engine to boost their performance and to reduce the deployment costs. Examples include the Amazon EC2 cloud platform that opens a dedicated interface for BitTorrent, and LeafWeb P2P gaming platform that allows users to invite others from their Facebook profiles to play online games.

Yet there remain a number of challenges for P2P to be a fundamental infrastructure service for pervasive Internet applications: It is necessary to deal with peer churns to provide stable and robust quality-of-service; the inventiveness of individual users and that of Internet Service Providers (ISPs)

\footnotetext{
J. Liu $(\bowtie)$

Simon Fraser University, Burnaby, Canada

e-mail: jcliu@sfu.ca

K. Xu

Tsinghua University, Beijing, China

e-mail: xuke@tsinghua.edu.cn

\section{Y. Xiong}

Microsoft Research Asia, Beijing, China

e-mail: yqx@microsoft.com

D. Ma

North China University of Technology, Beijing, China

e-mail: madongchao@gmail.com

\section{K. Shuang}

Beijing University of Posts and Telecommunications, Beijing, China

e-mail: shuangk@bupt.edu.cn

have to be carefully addressed for incorporating a P2P engine; and security issues have been brought to the front end as substantial P2P based attacks, viruses and botnets appear. These problems can be even more severe with the emerging new applications.

The objective of this special issue is to summarize the recent research and development toward making P2P a better infrastructure service. After a thorough review process, ten papers were accepted after revision and a second round of review (some with a third round of review).

The first set of papers discuss the traffic management and content sharing in peer-to-peer systems.

Paper "On the Collaboration of Different Peer-to-Peer Traffic Management Schemas" by Xin Liu, Haiyang Wang, and Lei Zhangaims to solve the pressure on the ISPs from P2P traffic. Different with the existing schemes that only use caching or redirection, they combines the two methods together with a coherent framework, Tod-Cache (Traffic Orientated Distributed Caching).

Paper "The Performance and Locality Tradeoff in BitTorrent-like File Sharing Systems" by Wei Huang, Chuan $\mathrm{Wu}$, Zongpeng $\mathrm{Li}$, and Francis C.M. Lau characterizes the performance and locality tradeoff in P2P as a multi-objective b-matching optimization problem and designs fully distributed peer selection algorithms.

Paper "BufferBank: A Distributed Cache Infrastructure For Peer-to-peer Application" by Huang Bin, Sun Zhigang, Chen Hongyi, Mao Jianbiao, Zhang Ziwen proposes a distributed P2P caching infrastructure named BufferBank in the edge of network. This infrastructure uses the memory of users at the edge network as the cache space and the authors also provide a number of application interfaces for P2P applications to use the cache space.

Paper "A Scalable P2P Overlay Based on Arrangement Graph with Minimized Overhead" by Ssu-Hsuan Lu, KuanChing Li, Kuan-Chou Lai, and Yeh-Ching Chung examines 
the scalability and exibility of P2P systems and presents an arrangement graph to form a $\mathrm{P} 2 \mathrm{P}$ overlay, so as to reduce system overhead and bind routing hops.

Paper "A research on dynamic allocation of network resources based on P2P traffic planning" by Dongchao Ma, Xiaoliang Wang, Wenlong Chen, Shen Yang, and Li Ma analyzes the current mainstream P2P optimization strategies and put forward a resources dynamic allocation technology based on the deployment of P2P traffic which stand on the operator point of view.

The second set of papers discuss the new trends and challenges in peer-to-peer systems, including social networking, incentive, and security.

Paper "Social Trust: Enabling Long-term Social Cooperation in P2P Services" by Yusuo Hu, Danqi Wang, HuiZhong, and Feng $\mathrm{Wu}$, design and implement Social Trust that is a social P2P network based on peers' common interests. The paper also proposes a distributed trust mechanism that reflects peer's cooperation level and serves as the credit limit between them. The proposed distributed trust mechanism is proved to be secure and can defend against various forms of attacks.

Paper "Improving Sustainability of BitTorrentDarknets" by Xiaowei Chen, Xiaowen Chu, and Zongpeng Li brings forth new ideas about "Poor Downloading Motivation" problem in private $\mathrm{P} 2 \mathrm{P}$ communities and proposes a Predator-prey model and queuing model to optimize the SLR range.

Paper "A Comprehensive Study of the Use of Advertisements as Incentives in P2P Streaming Systems" by Bo-Chun Wang, Alix L.H. Chow, LeanaGolubchik addresses the problem of free-riding in peer-to-peer media streaming by proposing the use of advertisements as an incentive for peers to contribute upload capacity.

Paper "An Efficient ECC-Based Mechanism for Securing Network Coding-Based P2P Content Distribution" by Ruixuan Li, Heng He, Zhiyong Xu and Weijun Xiao proposes an efficient ECC-based mechanism for securing network coding-based P2P content distribution including an efficient network coding signature scheme and an identity-based malicious peer identification scheme. Security analysis demonstrates that proposed approach can resist hash collision attacks, signature forgery attacks, and collusion attacks with arbitrary number of colluding malicious peers.

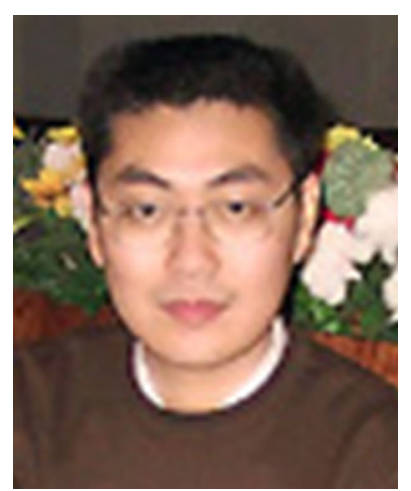

Jiangchuan Liu (S'01-M'03$\mathrm{SM}^{\prime} 08$ ) received the BEng degree (cum laude) from Tsinghua University, Beijing, China, in 1999, and the PhD degree from The Hong Kong University of Science and Technology in 2003, both in computer science. $\mathrm{He}$ is a recipient of Microsoft Research Fellowship (2000), Hong Kong Young Scientist Award (2003), and Canada NSERC DAS Award (2009). He is a co-recipient of the Best Student Paper Award of IWQoS'2008, the Best Paper Award (2009) of IEEE ComSoc Multimedia Communications Technical Committee, and Canada BCNet Broadband Challenge Winner Award 2009. He is currently an Associate Professor in the School of Computing Science, Simon Fraser University, British Columbia, Canada, and was an Assistant Professor in the Department of Computer Science and Engineering at The Chinese University of Hong Kong from 2003 to 2004. His research interests include multimedia systems and networks, wireless ad hoc and sensor networks, and peer-to-peer and overlay networks. He is a Senior Member of IEEE and a member of Sigma Xi. He is an Associate Editor of IEEE Transactions on Multimedia, and an editor of IEEE Communications Surveys and Tutorials. He is TPC Vice Chair for Information Systems of IEEE INFOCOM'2011.

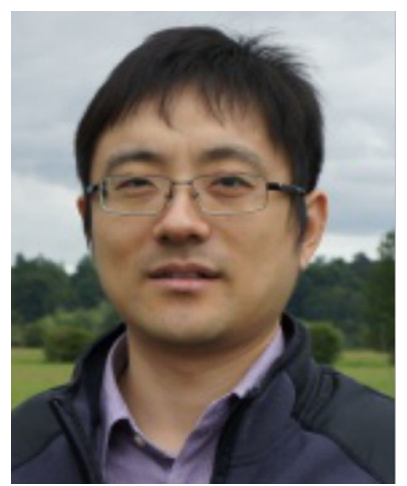

Ke Xu (M'02-SM'09) received his Ph.D. from the Department of Computer Science of Tsinghua University. He is currently a full professor in the Department of Computer Science of Tsinghua University. He has published more than 100 technical papers and holds 20 patents in the research areas of next generation Internet, P2P systems, Internet of Things ( Io T), network virtualization and optimization. $\mathrm{He}$ is a member of ACM. He has guest edited several special issues

in IEEE and Springer Journals. He is serving as associate editor of Springer journal Networking Science. Currently, he is holding visiting professor position of University of Essex. 


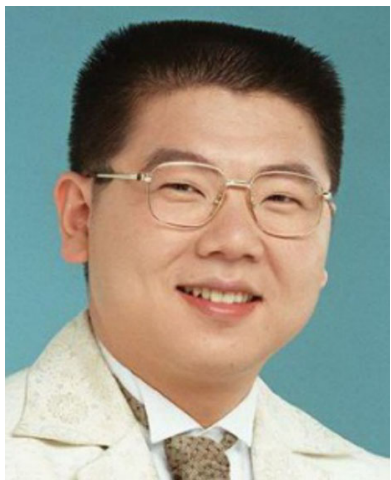

Yongqiang Xiong is now with Wireless and Networking Group at Microsoft Research Asia as a lead researcher. He received his B.S., M.S., and Ph.D degrees from Tsinghua University, Beijing, China in 1996, 1998 and 2001 , respectively, all in computer science. His research interests include cata center networking, peer-to-peer networking, routing protocols for both MANETs and overlay networks, and network security. He has published over 30 papers, and served as TPC member or reviewers for the international key conferences and leading journals in the areas of wireless and networking. Dr. Xiong is member of IEEE.

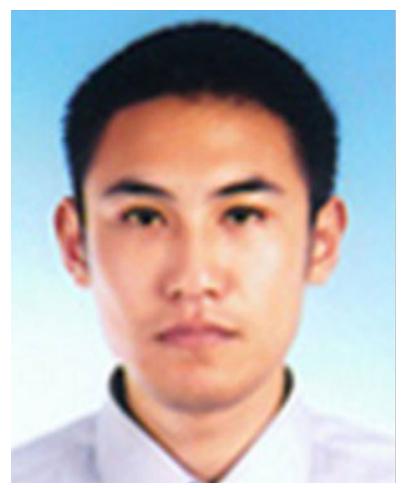

Dongchao Ma born in 1980, Ph.D., associate professor, Information Engineering Institute, North China University of Technology. His research interests include Next Generation Internet, network management and traffic flow management.

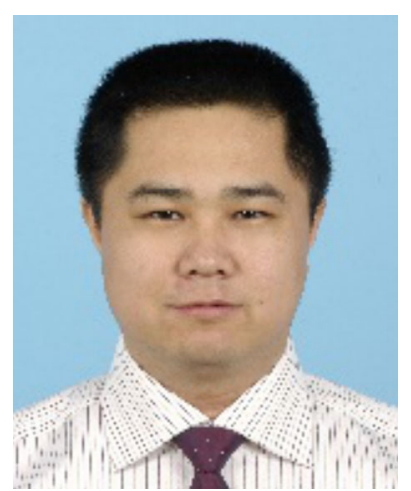

Kai Shuang is with State Key Laboratory of Networking \& Switching Technology. Beijing University of Posts \&Telecommunications (BUPT). 